\title{
Wood zone plate fishnet metalens
}

\author{
Bakhtiyar Orazbayev $^{1, *}$, Miguel Beruete ${ }^{1,2}$, and Miguel Navarro-Cía ${ }^{3,4}$ \\ 1 Antennas Group-TERALAB, Universidad Pública de Navarra, Campus Arrosadía, 31006 Pamplona, Spain \\ 2 Institute of Smart Cities, Universidad Pública de Navarra, Campus Arrosadía, 31006 Pamplona, Spain \\ 3 Optical and Semiconductor Devices Group, Department of Electrical and Electronic Engineering, Imperial College London, \\ London SW7 2BT, UK \\ 4 School of Physics and Astronomy, University of Birmingham, Birmingham B15 2TT, UK
}

Received 14 September 2015 / Accepted 12 November 2015

\begin{abstract}
Fresnel-zone plate lenses provide focusing performance while having low profile. Unfortunately, they usually display higher reflection losses than conventional dielectric lenses. Here, we demonstrate a low-profile Wood zone plate metalens based on the fishnet metamaterial working in a near-zero regime with an equivalent refractive index less than unity $\left(n_{f}=0.51\right)$. The metalens is made of alternating dielectric and fishnet metamaterial concentric rings. The use of fishnet metamaterial allows reducing the reflections from the lens, while maintaining low profile, low cost and ease of manufacturing. The lens is designed to work at the W-band of the millimeter-waves range with a focal length $F L=22.8 \mathrm{~mm}\left(7.5 \lambda_{0}\right)$ aiming at antenna or radar system applications. The focusing performance of the lens along with its radiation characteristics in a lens antenna configuration have been studied numerically and confirmed experimentally, showing a gain improvement of $\sim 2.5 \mathrm{~dB}$ with respect to a fishnet Soret metalens.
\end{abstract}

Key words: Fishnet metamaterial, Fresnel zone, Lens.

\section{Introduction}

The Wood-type or phase corrected zone plate is a member of the Fresnel-zone plate lens (FZPL) family and is free from the main drawback of the Soret-type lenses - high reflections from the even (odd) zones, which are made of opaque rings $[1,2]$. It is done by reversing the phase of the wave in these zones instead of blocking them. This idea was first suggested by Rayleigh [3] and implemented by Wood [4]. Hence, the zone plate with different phase retardation for odd (even) and even (odd) zones is also known as Rayleigh-Wood lens or Wood zone plate lens (WZPL) and outperforms the Soret lens in diffraction efficiency. However, the thickness of the WZPL depends on the difference between the refractive indices of the materials used for even and odd zones, and for materials with close values of refractive index the lens becomes too thick and heavy. Conversely, for high values of the refractive index the transmission coefficient decreases rapidly, reducing the focusing efficiency of the lens $[1,2]$.

One possible way to improve the focusing efficiency while maintaining the low profile and light weight is to apply a

*e-mail: b.orazbayev@unavarra.es metamaterial for even or odd zones. Metamaterials, descendants of the artificial dielectrics [5], enable the engineering of extreme values of refractive index, unattainable with natural materials, while tailoring simultaneously the effective impedance [6]. The latter avoids insertion losses due to reflection, which results in increasing overall efficiency in the case of lenses. The negative index of refraction permits reducing significantly the thickness of the WZPL, since the phase difference of $\pi$ between odd and even zones can be obtained with small thickness $\left(<\lambda_{0} / 4\right)$.

A promising metamaterial for microwave and millimeter wave range is a fishnet metamaterial, which consists of closely-packed subwavelength hole arrays working under extraordinary optical transmission [7-9]. Such metamaterial has been used for advanced lens design and its good performance has been confirmed in previous works [10-17]. Due to the leaky-wave mechanism present in the fishnet metamaterial, it has the potential to lower insertion loss $[8,16]$.

In this work we propose a hybrid half-wavelength WZP fishnet metalens with enhanced matching with free space. This hybrid design utilizes the advantages of the fishnet metamaterial to overcome the drawbacks of the classical WZPL. The proposed WZP fishnet metalens has been designed, fabricated 
and experimentally analyzed at the W-band of the millimeterwave range. The experimental results of the radiation pattern show a gain of $16.6 \mathrm{~dB}$ at the operation frequency $f=99 \mathrm{GHz}$ $\left(\lambda_{0}=3.03 \mathrm{~mm}\right)$. These results are supported by numerical simulations.

\section{Design}

\subsection{Fishnet metamaterial dispersion}

To create different optical paths in even and odd zones we use two materials: a standard dielectric (from a commercial substrate) and a fishnet metamaterial, which has a dispersive nature and whose index of refraction can be adjusted by varying, for instance, the separation between the plates $d_{z}$ and the diameter $a$ of the subwavelength holes (see inset of Figure 1a). In this work we use the commercial substrate Rogers RO5880 ${ }^{\mathrm{TM}}$, with thickness $t_{d}=0.381 \mathrm{~mm}$, dielectric permittivity $\varepsilon_{r}=2.2$ and loss tangent $\tan \delta=9 \times 10^{-4}$. The unit cell of the fishnet metamaterial (inset of Figure 1a) has the following dimensions: $d_{x}=1.26 \mathrm{~mm}, d_{y}=2.1 \mathrm{~mm}, d_{z}=0.398 \mathrm{~mm}$ which corresponds to a metal thickness $t_{m}=0.017 \mathrm{~mm}$ (yellow layer in the inset of Figure 1a) and a dielectric thickness of dielectric $t_{d}=0.381 \mathrm{~mm}$ (semitransparent blue layer in the inset of Figure 1a), and variable hole diameter $a$. The equivalent refractive index for a finite number of plates varies with the number of periods due to the inhomogeneity of the fishnet metamaterial [18-20]. Considering our previous experience we use four plates (plus one dielectric plate as a protection cover), since it is a good trade-off between total thickness and electromagnetic performance in terms of insertion loss [10-16]. Therefore, the total thickness of the structure is $w=4 t_{m}+5 t_{d}=1.97 \mathrm{~mm}\left(\sim 0.62 \lambda_{0}\right)$. From the equation of the thickness of the WZPL $w=\lambda_{0} /\left(\sqrt{ } \varepsilon_{r}-\sqrt{ } \varepsilon_{f}\right)$ (where $\varepsilon_{f}$ is the equivalent permittivity of the fishnet metamaterial) we find the required refractive index $n_{f}=0.51$ of the fishnet metamaterial for the given thickness of the metalens. Here we should note that the equivalent refractive index $n_{f}<1$ allows to decrease the total thickness of the metalens $w$.

The equivalent refractive index of the finite fishnet metamaterial was obtained using the transient solver of CST Microwave Studio ${ }^{\mathrm{TM}}$ in the range $85-110 \mathrm{GHz}$. Periodic boundary conditions were used and the metal was modelled as copper $\left(\sigma_{\mathrm{Cu}}=5.96 \times 10^{7} \mathrm{~S} / \mathrm{m}\right)$. A fine hexahedral mesh was used, with minimum and maximum edge lengths of $0.007 \mathrm{~mm}$ $\left(\sim 0.003 \lambda_{0}\right)$ and $0.64 \mathrm{~mm}\left(\sim 0.2 \lambda_{0}\right)$, respectively. The equivalent index of refraction for four plates was calculated as $n_{m}=\Delta \varphi /\left(k_{0} \Delta d\right)$, where $\Delta \varphi$ is the phase variation along the total thickness, $\Delta d$, and $k_{0}$ is the wave number in free-space. The resulting color-map for equivalent index of refraction for four cascaded plates as a function of frequency and radius of the hole is shown in Figure 1a. Also it is important to ensure a high transmission coefficient at the design frequency. To this end the transmission coefficient $S_{21}$ was obtained as a function of frequency and radius of the holes and is plotted in Figure $1 \mathrm{~b}$. From these color-maps we chose $99 \mathrm{GHz}\left(\lambda_{0} \sim 3.03 \mathrm{~mm}\right)$ as the operation frequency and $a=1.08 \mathrm{~mm}$ as the diameter of the holes where the index of refraction is $n_{f}=0.51$.
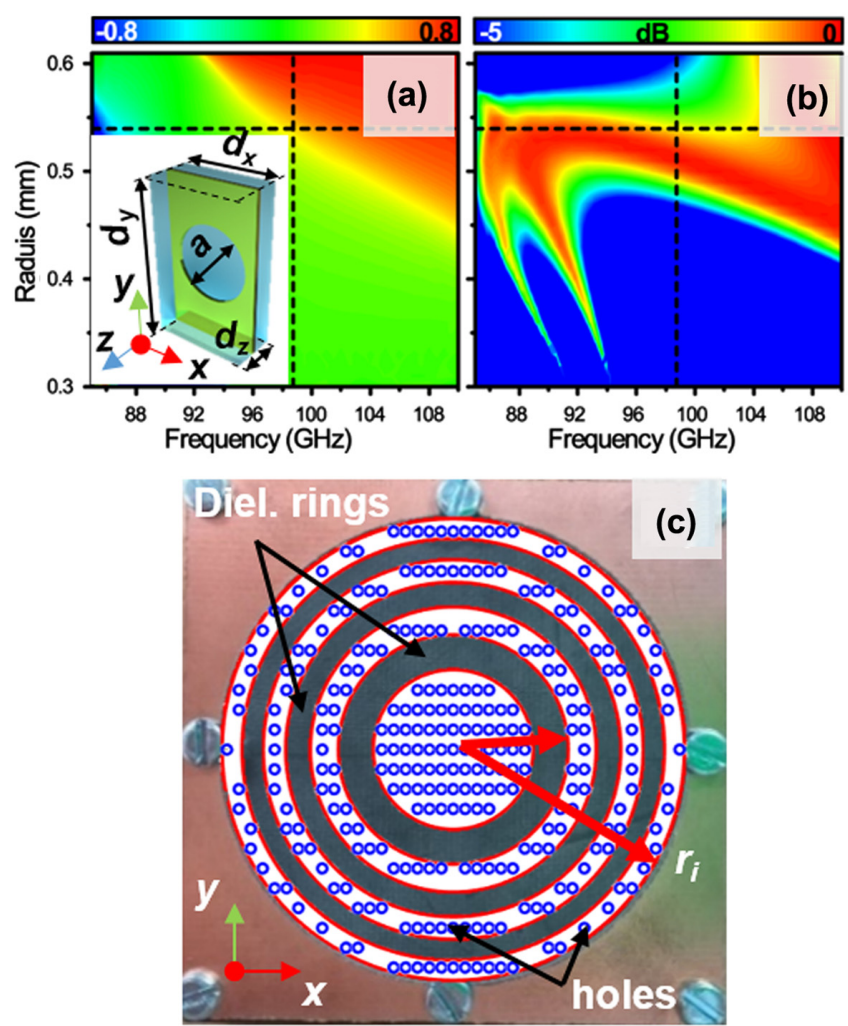

Figure 1. (a) Equivalent refractive index, $n_{f}$, for a fishnet metamaterial made of four plates. Vertical and horizontal black dashed lines correspond to the working frequency $f_{0}=99 \mathrm{GHz}$ and hole diameter $a=0.54$ respectively. Parameter definition of fishnet metamaterial unit cell (Inset); (b) Transmission coefficient $S_{21}$ for a four-plate fishnet metamaterial; (c) Fabricated WZP fishnet metamaterial lens and its seven Fresnel zones.

\subsection{Fresnel zones}

The radii of the Fresnel zones $r_{i}$ of the WZPL can be calculated using the next design equation $[1,2]$ :

$$
\eta=\sqrt{\frac{2 F L \cdot \lambda_{0} \cdot i}{p}+\left(\frac{i \cdot \lambda_{0}}{p}\right)^{2}}
$$

where $F L$ is the focal length of the WZPL, $\lambda_{0}$ is the operation wavelength, $i$ is an integer denoting zone number and $p$ is the number of phase quantization levels. The performance of the WZPL can be improved by increasing the number of quantization levels, i.e. going from half-wavelength to multilevel zone plates [21]. However, given the comparable size of the fishnet in-plane periods $d_{x}$ and $d_{y}$ with the radii of the Fresnel zones, the maximum number of quantization levels is $p=2$. The total performance of the WZP fishnet metalens also depends on the filling ratio of the Fresnel zones, i.e. how well the zones are filled with holes. This, in turn, depends on multiple design parameters, such as $F L, \lambda_{0}, i$. For this reason, a three-dimensional analytical calculation was done initially by means of the Huygens-Fresnel principle for a fast prototyping.

After the optimization procedure the following parameters were chosen: $F L=22.8 \mathrm{~mm}\left(\sim 7.5 \lambda_{0}\right), i=7, p=2$. The final 
lens profile and fabricated WZP fishnet metalens is shown in Figure 1c. The radius of the last zone was $r_{7}=24.5 \mathrm{~mm}$. The positive (odd) zones were made of holes of the same dimension as the fishnet (see Figure 1a). Thus, the whole structure has dimensions of $50 \mathrm{~mm} \times 50 \mathrm{~mm} \times 1.973 \mathrm{~mm}$ along the $x$-, $y$ - and $z$-axis, respectively. The WZP fishnet metalens was fabricated using the commercial substrate Rogers RO5880 ${ }^{\mathrm{TM}}$ with thickness $t_{d}=0.381 \mathrm{~mm}$ coated with a copper layer of thickness $t_{m}=0.017$, by milling the holes and rings in the metal, keeping the dielectric substrate untouched. Then all layers and an additional protective outer layer were fastened with screws.

\section{Results}

\subsection{Analytical results}

The designed lens was analyzed using the Huygens-Fresnel principle. From this method the power distribution was calculated as a function of frequency and position along the optical axis of the lens (Figure 2a). The absolute maximum in this case occurs at $96 \mathrm{GHz}$, with focal length $F L_{1}=22.3 \mathrm{~mm}\left(\sim 7.4 \lambda_{0}\right)$. A secondary focus is observed at $F L_{2}=41 \mathrm{~mm}\left(\sim 13.5 \lambda_{0}\right)$. Like any diffractive optical element, the WZPL suffers from chromatic aberration which results in a shift of the focal point with frequency. Finally, the power distribution color-maps were calculated at the design frequency for both $x z$ - and $y z$-plane and presented in Figures $3 \mathrm{a}$ and $3 \mathrm{~b}$, respectively. In this figure clear foci can be observed with a transverse dimension $0.66 \lambda_{0}$ for both $x z$ - and $y z$-plane.

\subsection{Simulation results}

Next, a full-wave numerical analysis of the realistic 3D model of the fishnet WZPL was done using the transient solver of the CST Microwave Studio ${ }^{\mathrm{TM}}$. In the simulations, the metal copper layer was modelled using a lossy metal with the bulk conductivity of copper $\left(\sigma_{\mathrm{Cu}}=5.96 \times 10^{7} \mathrm{~S} / \mathrm{m}\right)$. A fine hexahedral mesh was used with minimum and maximum mesh cell sizes of $0.12 \mathrm{~mm}\left(\sim 0.04 \lambda_{0}\right)$ and $0.43 \mathrm{~mm}\left(\sim 0.14 \lambda_{0}\right)$, respectively. A vertically polarized $\left(E_{y}\right)$ plane wave with normal incidence was used to illuminate the lens. To emulate a lens in free space, perfectly matched layers were used at the boundaries of the simulation box. Given the two-fold symmetry of the problem, electric and magnetic symmetries were imposed in the $x z$-plane $(y=0)$ and $y z$-plane $(x=0)$, respectively, to reduce computation time. The simulation was run for a sufficiently long time to ensure steady-state regime so that the continuous-wave information computed by Fourier transformations was valid. The color-map of the power spectrum as a function of $z$ position was obtained by placing E-field and H-field probes along the optical axis (z-axis) with a $0.5 \mathrm{~mm}$ step. These probes record the waveform at their positions and by Fourier transformation the $E$ - and $H$-field spectra are obtained.

The transmitted power enhancement can be obtained as the ratio between the intensity with and without lens for each $z$ position: $E_{\text {enh }}=10 \log _{10}\left(P_{\text {lens }} / P_{\text {free }}\right)$, where $P_{\text {lens }}$ is the power received with the lens and $P_{\text {free }}$ is the power received when

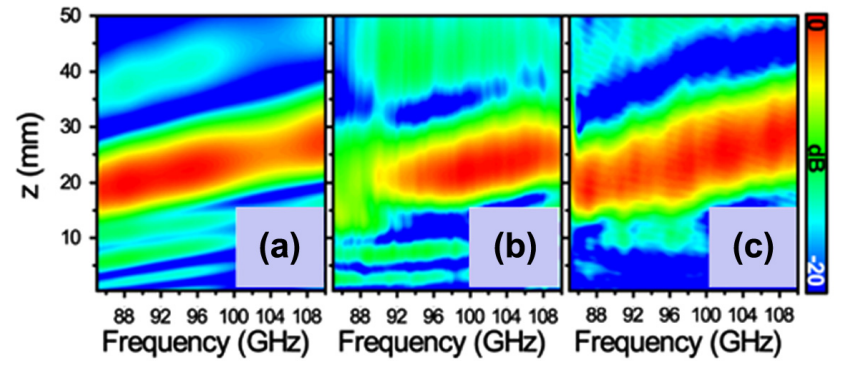

Figure 2. Normalized power distribution along $z$ axis for the frequency range 85-110 GHz: (a) analytical results, (b) simulation and (c) experimental results.

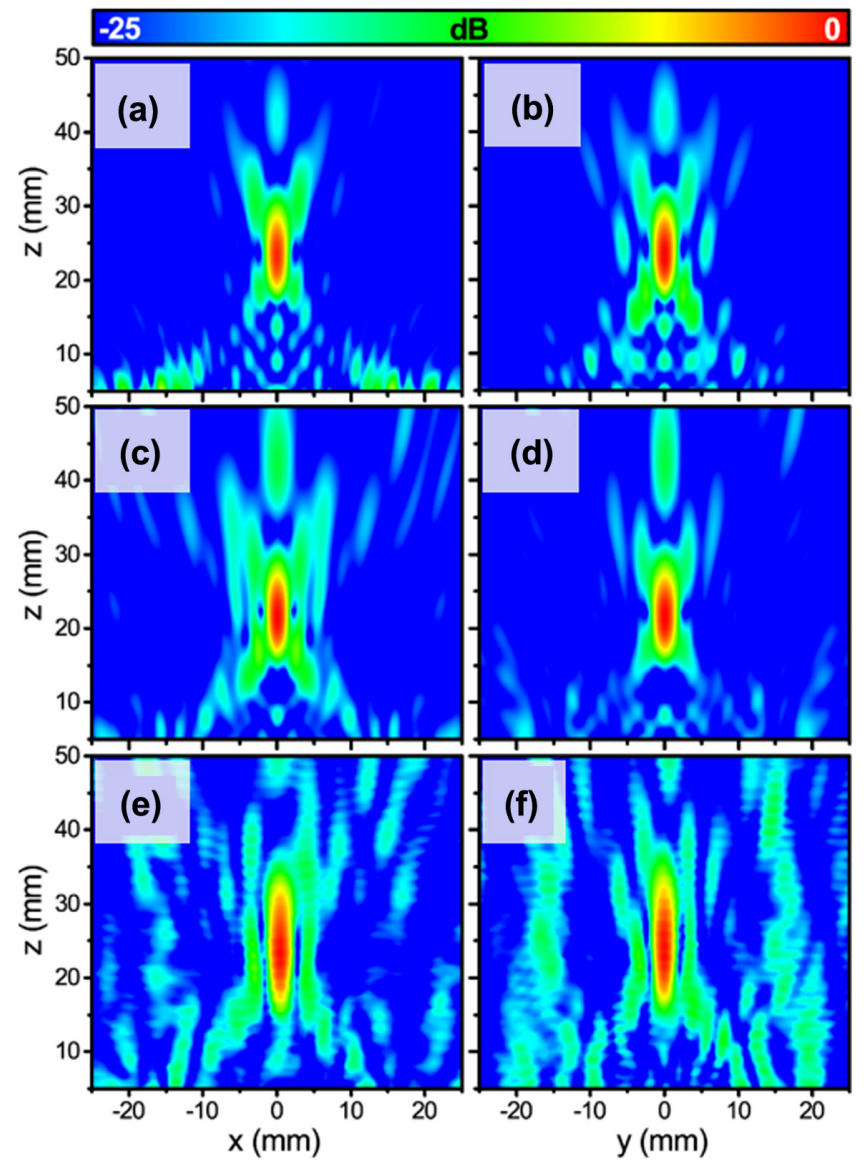

Figure 3. Normalized spatial power distribution on the $x z$-plane (left column) and $y z$-plane (right column) for: (a, b) analytical results at $99 \mathrm{GHz}$; (c, d) simulation results at $99 \mathrm{GHz}$; (e, f) experimental results at $99 \mathrm{GHz}$.

no lens is present. The obtained power spectrum is shown in Figure $2 \mathrm{~b}$. The focal point shifts within the frequency range 92-108 GHz, from $20 \mathrm{~mm}\left(6.6 \lambda_{0}\right)$ up to $25 \mathrm{~mm}\left(8.2 \lambda_{0}\right)$ along the $z$-axis. These results resemble the analytical results, with some differences. Firstly, a blueshift is noticeable. This arises from the fact that in the analytical model the dispersion of the fishnet metamaterial was not taken into account for the sake of simplicity. Secondly, the secondary focal spot in simulations is more pronounced. The power enhancement corresponding to the first maximum is $19 \mathrm{~dB}$ at $99 \mathrm{GHz}$ 


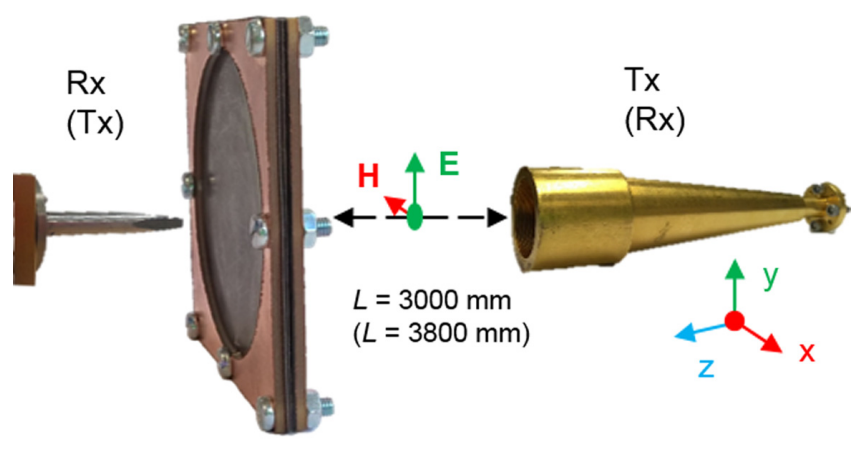

Figure 4. Scheme of experimental setup for the focal measurements (radiation measurements) with a waveguide probe as a receiver (transmitter) and a standard high gain horn antenna as a transmitter (receiver).

$\left(\lambda_{0} \sim 3.04 \mathrm{~mm}\right)$ with a focal distance $F L=22.5 \mathrm{~mm}$ $\left(\sim 7.4 \lambda_{0}\right)$. Finally, in Figures $3 \mathrm{c}$ and $3 \mathrm{~d}$ the power distribution at $99 \mathrm{GHz}$ is shown for $x z$ - and $y z$-planes respectively, with transverse dimension of the foci $0.66 \lambda_{0}$ for $x z$ - and $y z$-plane. These color-maps also match the analytical results with some minor, yet expected, differences.

\subsection{Experimental results}

The experimental characterization was done using an $\mathrm{AB}$ Millimetre $^{\mathrm{TM}}$ quasi-optical vector network analyzer with the setup illustrated in Figure 4. The metalens was illuminated with a $W$-band corrugated horn antenna, placed at distance $L=3000 \mathrm{~mm}$ from the lens. At this distance the radius beam waist of the Gaussian beam is $\sim 310 \mathrm{~mm}$, which ensures a uniform illumination of the lens. The $x z$ raster scanning was done using a waveguide probe WR-8.0 as a detector. Millimeterwave absorbers were used throughout the setup to mimic anechoic chamber conditions. First, the calibration was done by recording the transmitted power without the lens. Next, the power distribution as a function of frequency and $z$ position was measured by moving the detector from 5 to $50 \mathrm{~mm}$ away from the metalens along $z$-axis (with $0.5 \mathrm{~mm}$ step) while recording the spectrum in the range $85-110 \mathrm{GHz}$. The experimentally measured color-map for the power spectrum along the optical $z$-axis is shown in Figure 2c. The measurement results match our previous analytical and numerical results (Figures $2 \mathrm{a}$ and 2b). The maximum power enhancement $12.44 \mathrm{~dB}$ occurs at $97.75 \mathrm{GHz}$ with a focal distance $F L=23 \mathrm{~mm}\left(7.57 \lambda_{0}\right)$. Next, the spatial power distribution at this frequency was obtained experimentally for the $x z-, y z$ planes, see Figures $3 \mathrm{e}$ and $3 \mathrm{f}$. The qualitative agreement with the numerical results is evident. The transverse dimension of the foci is $0.69 \lambda_{0}$ for $x z-y z$-plane. To facilitate the comparison, all the results are gathered in Table 1.

\subsection{Lens antenna system}

Next we investigate the performance of fishnet WZPL in a lens-antenna configuration. For the numerical characterization, the simulations included the realistic waveguide probe WR-8.0 as a feeder, placed at the previously numerically-found focal position $z=22.5 \mathrm{~mm}$. The rest of the simulation parameters were those described previously. Far-field monitors were used to record the radiation pattern of the lens within the frequency range $85-110 \mathrm{GHz}$ with a step of $0.25 \mathrm{GHz}$

For the experimental verification, the waveguide probe WR-8.0 was used as a feeder placed at the experimental focal length $F L=23 \mathrm{~mm}\left(7.6 \lambda_{0}\right)$. A schematic of the experimental setup is shown in Figure 4. A high gain standard horn antenna was placed $3800 \mathrm{~mm}$ away from the flat face of the zoned lens to detect the radiated power. The radiation diagram was measured by rotating the feeder and metalens from $-90^{\circ}$ to $+90^{\circ}$ with $1^{\circ}$ step. Absorbing material was also used throughout the setup for reflection suppression.

Numerical results of co- and cross-polar angular power distributions for $E$ - and $H$-plane are displayed in Figures $5 \mathrm{a}$ and $5 \mathrm{~d}$. The maximum is located at $99 \mathrm{GHz}$, in agreement with

Table 1. Focal properties and radiation pattern parameters

\begin{tabular}{|c|c|c|c|c|c|c|c|c|c|c|c|}
\hline & Frequency, $\mathrm{GHz}$ & $\begin{array}{c}F L^{1}, \mathrm{~mm} \\
E \text {-plane } \\
H \text {-plane }\end{array}$ & \multicolumn{2}{|c|}{$\mathrm{FWHM}^{2}, \mathrm{~mm}$} & $\begin{array}{c}\mathrm{DF}^{3}, \mathrm{~mm} \\
E \text {-plane } \\
H \text {-plane }\end{array}$ & \multicolumn{2}{|c|}{$\mathrm{HPBW}^{4},{ }^{\circ}$} & \multicolumn{2}{|c|}{$\mathrm{FNBW}^{5},{ }^{\circ}$} & \multicolumn{2}{|c|}{$\mathrm{FSLL}^{6},^{\circ}$} \\
\hline Simulation & $\begin{array}{c}99 \\
\lambda_{0}=3.03 \mathrm{~mm}\end{array}$ & $\begin{array}{c}22.5 \\
7.4 \lambda_{0}\end{array}$ & $\begin{array}{c}2 \\
0.66 \lambda_{0}\end{array}$ & $\begin{array}{c}2 \\
0.66 \lambda_{0}\end{array}$ & $\begin{array}{c}7.8 \\
2.6 \lambda_{0}\end{array}$ & 4 & 3.6 & 14 & 10 & -25 & -19 \\
\hline
\end{tabular}

\footnotetext{
${ }^{1} \mathrm{FL}$ is the focal length.

2 FWHM is the full width at half maximum.

${ }^{3} \mathrm{DF}$ is the depth of focus.

${ }^{4} \mathrm{HPBW}$ is the half-power beam width.

${ }^{5}$ FNBW is the first null beam width.

${ }^{6}$ FSLL is the first side-lobe level.
} 

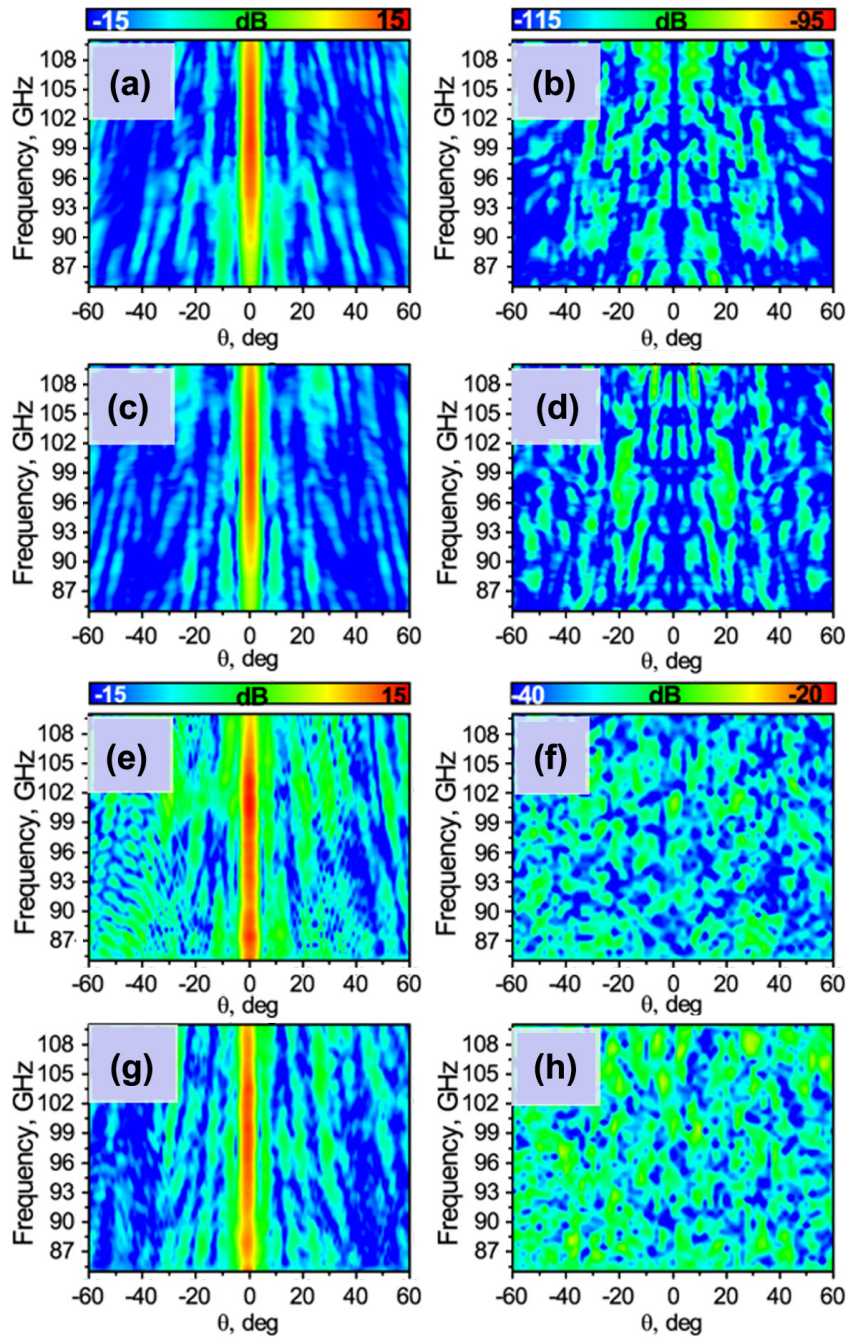

Figure 5. Numerical $(\mathrm{a}-\mathrm{d})$ experimental $(\mathrm{e}-\mathrm{h})$ radiation pattern of the WZP fishnet metalens antenna in the frequency range 85-110 GHz: co- (a, c, e, g) and cross-polarization (b, d, f, h). (a, b, e, f) E-plane and (c, d, g, h) $H$-plane.

the previous numerical study. The normalized experimental results for co- and cross-polar components as a function of frequency and angle are shown in Figures $5 \mathrm{e}$ and $5 \mathrm{f} E$-plane and Figures $5 \mathrm{~g}-5 \mathrm{~h} \mathrm{H}$-plane. The experimental maximum is located at $99 \mathrm{GHz}$. In Figures $6 \mathrm{a}$ and $6 \mathrm{~b}$ to facilitate the comparison, the normalized simulation and experimental radiation patterns for the $E$ - and $H$-plane are presented. The cross-polar components for the numerical results are omitted due to their negligible values. One can see that the radiation patterns for the main lobes are in good agreement, whereas side lobes are higher in the experiment. This is likely due to the fact that the waveguide WR-8.0 probe and horn antenna are not modelled. That is, the transmitter and receiver have not been de-embedded from the experimental results. Moreover, due to the several factors (discussed above) the experimental ratio main/sidelobe is lower than numerical, which results in higher lobes in normalized radiation patterns.

To complete the study, the numerical and experimental gain for the WZP fishnet metalens antenna is presented in
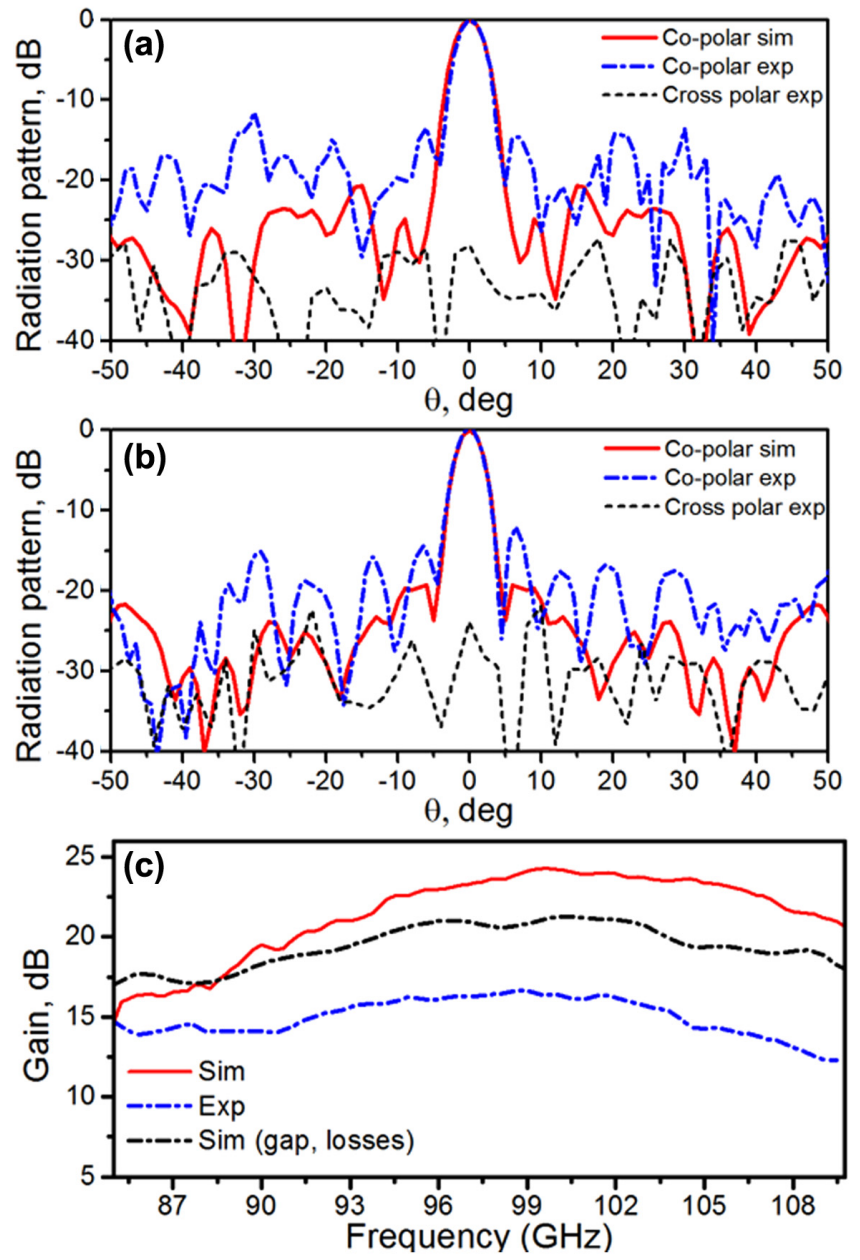

Figure 6. Normalized radiation patterns for: (a) E-plane and (b) $H$-plane at frequency $99 \mathrm{GHz}$ (simulation and experimental). (c) Simulation gain (solid red line), experimental gain (dash dotted blue line) and simulation gain, considering air gaps, larger diameter $a$ of subwavelength holes and losses (dash dotted black line).

Figure $6 \mathrm{c}$. In the experiment, the gain was obtained by comparing our lens antenna with a standard horn antenna following the gain comparison method [21]. To this end, first, the power enhancement $P_{\text {enh_lens }}=10 \log _{10}\left(P_{\text {lens }} / P_{\text {free }}\right)$ of the lensantenna was recorded, then it was replaced by a standard high gain horn antenna with gain $G_{\text {horn }}=22 \mathrm{~dB}$ and the transmitted power enhancement $P_{\text {enh_horn }}=10 \log _{10}\left(P_{\text {horn }} / P_{\text {free }}\right)$ was measured again. Thus the gain of the metalens-antenna can be found as: $G_{\text {lens }}=P_{\text {enh_lens }}-P_{\text {enh_horn }}+G_{\text {horn }}$. A high gain of $16.6 \mathrm{~dB}$ is found experimentally at $99 \mathrm{GHz}$ (solid red line). The numerical value of $24.3 \mathrm{~dB}$ is found at $99 \mathrm{GHz}$ by using the software-implemented far-field monitors (dashed blue line). The lower values of gain in the experiment can be explained as a result of different factors, such as experimental errors (misalignment, accuracy of distance measurement) and by defects in the fabrication (non-perfect contact between dielectric and metallic plates, errors in the radius of the holes) and effective substrate losses higher than nominal values. In order to confirm these factors, additional simulations for the complete 3D model of the metalens-antenna were run with diameter of holes 
$a=1.2 \mathrm{~mm}$, an air gap of $80 \mu \mathrm{m}$ and higher dielectric loss $\operatorname{tangent} \tan \delta=0.015$. The resulting gain is shown in Figure 6c (dashed dotted black line). An error in the diameter of the holes along with an air gap of $80 \mu \mathrm{m}$ between metallic and dielectric plates changes the effective permittivity of the fishnet metamaterial and therefore the phase at the output of each hole. This leads to the non-optimal phase difference (which is $\pi$ in the ideal case) between the odd and even rings, therefore to lower gain and frequency shift of the maximum. Consequently, the numerical gain of the lens with higher losses, error in the radius of the holes and air gap is closer to the experimental gain. To facilitate the comparison, all the results are gathered in Table 1.

\section{Conclusions}

In this paper, a comprehensive analysis of the WZP fishnet metalens has been performed. The study demonstrates the possibility of improving focusing and radiation performance of the phase-corrected lenses by applying the metamaterials. The experimental results show a good performance of the WZP fishnet metalens and are in good agreement with analytical and simulations results. The proposed metalens has low-profile design, relatively low cost and ease in manufacturing. Such compact device can find applications in wireless and radar system applications.

Acknowledgements. This work was sponsored by Spanish Government under contract TEC2014-51902-C2-2-R. B. O. is sponsored by Spanish Ministerio de Economía y Competitividad under grant FPI BES-2012-054909. M. B. is sponsored by the Spanish Government via RYC-2011-08221. M. N.-C. is supported by the Birmingham Fellowship. Authors are grateful to V. Pacheco-Peña for fruitful discussions and to G. Crespo for sample fabrication.

\section{References}

1. H.D. Hristov, Fresnel Zones in Wireless Links, Zone Plate Lenses and Antennas, Artech House, Norwood, MA, 2000.

2. I.V. Minin, O. Minin, Diffractional optics of millimetre waves, CRC Press, Bristol, 2004.

3. L. Rayleigh, Scientific papers, Cambridge Univ. Press 111 (1902) 87.

4. R.W. Wood, Phase-reversal zone-plates, and diffractiontelescopes, Philos. Mag. Ser. 545 (1898) 511.

5. R.E. Collin, Field theory of guided waves (2nd Ed.) IEEE Press, New York, 1991.

6. R. Marqués, F. Martín, M. Sorolla, Metamaterials with negative parameter: theory, design, and microwave applications, John Wiley \& Sons, New York, 2008.
7. T.W. Ebbesen, H.J. Lezec, H.F. Ghaemi, T. Thio, P.A. Wolff, Extraordinary optical transmission through sub-wavelength hole arrays, Nature 391 (1998) 667-669.

8. S. Zhang, W. Fan, N. Panoiu, K. Malloy, R. Osgood, S. Brueck, Experimental demonstration of near-infrared negative-index metamaterials, Phys. Rev. Lett. 95 (2005) 137404.

9. T. Matsui, A. Agrawal, A. Nahata, Z.V. Vardeny, Transmission resonances through aperiodic arrays of subwavelength apertures, Nature 446 (2007) 517-521.

10. M. Beruete, M. Navarro-Cía, M. Sorolla Ayza, I. Campillo, Planoconcave lens by negative refraction of stacked subwavelength hole arrays, Opt. Express 16 (2008) 9677-9683.

11. M. Navarro-Cía, M. Beruete, I. Campillo, M. Sorolla, Enhanced lens by $\varepsilon$ and $\mu$ near-zero metamaterial boosted by extraordinary optical transmission, Phys. Rev. B - Condens. Matter Mater. Phys. 83 (2011) 115112.

12. M. Navarro-Cía, M. Beruete, I. Campillo, M. Sorolla Ayza, Beamforming by left-handed extraordinary transmission metamaterial bi- and plano-concave lens at millimeter-waves, IEEE Trans. Antennas Propag. 59 (2011) 2141-2151.

13. V. Pacheco-Peña, B. Orazbayev, V. Torres, M. Beruete, M. Navarro-Cía, Ultra-compact planoconcave zoned metallic lens based on the fishnet metamaterial, Appl. Phys. Lett. 103 (2013), 183507.

14. V. Pacheco-Peña, B. Orazbayev, U. Beaskoetxea, M. Beruete, M. Navarro-Cía, Zoned near-zero refractive index fishnet lens antenna: steering millimeter waves, J. Appl. Phys. 115 (2014), 124902-1-8.

15. B. Orazbayev, V. Pacheco-Peña, M. Beruete, M. Navarro-Cía, Exploiting the dispersion of the double-negative-index fishnet metamaterial to create a broadband low-profile metallic lens, Opt. Express 23 (2015), 8555.

16. B. Orazbayev, M. Beruete, V. Pacheco-Peña, G. Crespo, J. Teniente, M. Navarro-Cía, Soret fishnet metalens antenna, Sci. Rep. 4 (2015), 9988.

17. V. Pacheco-Peña, M. Navarro-Cía, B. Orazbayev, I.V. Minin, O.V. Minin, M. Beruete, Zoned fishnet lens antenna with reference phase for side lobe reduction, IEEE Trans. Antennas Propag. 63 (2015), 3710-3714.

18. J. Zhou, T. Koschny, M. Kafesaki, C.M. Soukoulis, Negative refractive index response of weakly and strongly coupled optical metamaterials, Phys. Rev. B - Condens. Matter Mater. Phys. 80 (2009) 1-6.

19. M. Navarro-Cía, M. Beruete, M. Sorolla Ayza, I. Campillo, Viability of focusing effect by left-handed stacked subwavelength hole arrays, Phys. B Condens. Matter 405 (2010), 2950-2954.

20. M. Navarro-Cía, M. Beruete, I. Campillo, M. Sorolla Ayza, Fresh metamaterials ideas for metallic lenses, Metamaterials 4 (2010), 119-126.

21. G.A. Hurd, IEEE standard test procedures for antennas, Electron. Power, 26 (1980) 749.

Cite this article as: Orazbayev B, Beruete M \& Navarro-Cía M: Wood zone plate fishnet metalens. EPJ Appl. Metamat. $2015,2,8$. 